Nonlinear Dynamics manuscript No.

(will be inserted by the editor)

\title{
Duffing revisited: Phase-shift control and internal resonance in self-sustained oscillators
}

\author{
Sebastián I. Arroyo • Damián H. Zanette
}

Received: date / Accepted: date

\begin{abstract}
We address two aspects of the dynamics of the forced Duffing oscillator which are relevant to the technology of micromechanical devices and, at the same time, have intrinsic significance to the field of nonlinear oscillating systems. First, we study the stability of periodic motion when the phase shift between the external force and the oscillation is controlled -contrary to the standard case, where the control parameter is the frequency of the force. Phase-shift control is the operational configuration under which self-sustained oscillators -and, in par-

S. I. Arroyo

Consejo Nacional de Investigaciones Científicas y Técnicas, Universidad Nacional de Quilmes, Roque Sáenz Peña 352, 1876 Bernal, Buenos Aires, Argentina

E-mail: seba.arroyo7@gmail.com

D. H. Zanette

Consejo Nacional de Investigaciones Científicas y Técnicas, Centro Atómico Bariloche and Instituto Balseiro, 8400 San Carlos de Bariloche, Río Negro, Argentina

Tel.: +54-294-4445173

Fax: +54-294-4445299

E-mail: zanette@cab.cnea.gov.ar
\end{abstract}


ticular, micromechanical oscillators- provide a frequency reference useful for time keeping. We show that, contrary to the standard forced Duffing oscillator, under phase-shift control oscillations are stable over the whole resonance curve. Second, we analyze a model for the internal resonance between the main Duffing oscillation mode and a higher-harmonic mode of a vibrating solid bar clamped at its two ends. We focus on the stabilization of the oscillation frequency when the resonance takes place, and present preliminary experimental results that illustrate the phenomenon. This synchronization process has been proposed to counteract the undesirable frequency-amplitude interdependence in nonlinear time-keeping micromechanical devices.

Keywords Duffing oscillator · synchronization · micromechanical devices

\section{Introduction}

Since the 1970s, the Duffing oscillator -first introduced by the German engineer Georg Duffing in 1918 [1] - has been repeatedly invoked as a paradigm for nonlinear behavior in classical mechanical systems [2]. The mere addition of a cubic force to a linear damped oscillator deploys a plethora of complex phenomena, but still preserves the simple mathematical form and low dimensionality that makes the system readily amenable to computational exploration and, to a certain extent, to analytical study. Under the action of an external harmonic force, the Duffing oscillator's response can be bistable, with two possible oscillation amplitudes for each frequency. This property, which is directly related to the well-known Duffing leaning resonance curve [3], has been exploited as an illustration of catastrophe theory [4]. If, moreover, the sign of the linear force is inverted, creating a double- 
well potential, the dynamics can turn into non-periodic motion, with a strange attractor that became a traditional example of low-dimensional chaos [5, 6].

Much more recently, the Duffing oscillator acquired relevance in the realm of microtechnologies [7,8,9]. It has since long been known that the Duffing equation stands for the leading nonlinear correction to the oscillations of an elastic beam clamped at its two ends [10,11]. Minute vibrating silica beams, in turn, have been proposed as pacemakers for the design of time-keeping devices at the microscale, where traditional quartz crystals are difficult to build and operate [7]. Since, to overcome the effect of noise, these microscopic silica beams must vibrate at relatively large amplitudes, their oscillations take place within the nonlinear regime and -in clamped-clamped configurations [8] - the appropriate mathematical description is thus given by the Duffing equation.

In order to function as a pacemaker, a mechanical system must be able to sustain stationary periodic motion with an autonomously generated frequency. This can be achieved in practice by inserting the oscillator into a feedback electronic circuit 12 which, first, reads the signal generated by the oscillator's displacement from equilibrium. This signal is then conditioned by shifting its phase by a prescribed amount (or, equivalently, by inserting a time delay) and fixing its amplitude. Once conditioned, the signal is transformed into a mechanical force and reinjected to act on the oscillator. The oscillator, in turn, responds to this action as an ordinary resonator, except that the "external" force possesses the frequency generated by the oscillator itself. The result of this feedback process, schematized in Fig. 1] is that the mechanical system reaches self-sustained periodic motion, with the only external input of the power needed to condition the electric signal. The frequency of the oscillations is determined by the mechanical properties 


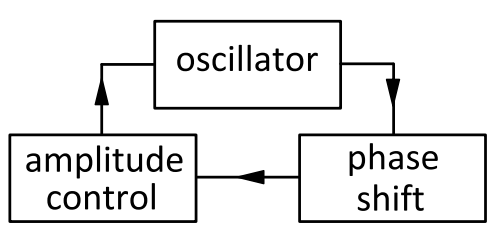

Fig. 1: Schematic representation of the feedback process that stirs self-sustained oscillations. The electric signal produced by the oscillatory motion is conditioned by shifting its phase and adjusting its amplitude, and is then reinjected as a mechanical force acting on the oscillator itself.

of the oscillator and the parameters of signal conditioning. From the dynamical viewpoint, this self-sustained configuration has the interest that the control parameter, to which the experimenter has access at the conditioning stage, is the phase shift between the oscillation and the force -instead of the frequency, as in the standard case of an externally forced oscillator.

In this paper, we focus on two aspects of the dynamics of the self-sustained Duffing oscillator which, apart from their interest from a theoretical perspective, have specific implications in the technological applications of the system. In both cases, we provide approximate analytical results and a numerical validation. After briefly reviewing the mathematical model and its main properties in Section 2, we first analyze the stability of oscillatory motion under controlling the phase shift between the oscillations and the self-sustaining force (Section 3). In contrast with the case where the frequency is controlled, where both stable and unstable solutions are found for a given set of parameters, we find that in our case oscillations are always stable. Then, in Section 4, we analyze a model for the coupling between the main oscillation mode and a higher-harmonic linear mode in a clamped-clamped oscillator. The ensuing internal resonance, with mutual synchronization of the 
two modes, has been invoked as a possible method to neutralize the undesirable dependence of the frequency on the amplitude, characteristic of any nonlinear oscillating system [8]. We present an experimental demonstration of the internal resonance under phase-shift control, and fit the experimental results with a simple analytical approximation to the model. Our conclusions are drawn in Section 5.

\section{The self-sustained Duffing oscillator}

In its main oscillation mode, an elastic beam clamped at its two ends vibrates transversally, much like a plucked string [8]. For moderate amplitudes, the displacement from equilibrium is well described by a coordinate $x(t)$ satisfying

$$
\ddot{x}+Q^{-1} \dot{x}+x+\beta x^{3}=f_{0} \cos \left(\phi+\phi_{0}\right) .
$$

This equation of motion has been normalized by the effective mass, and time units have been chosen in such a way that the frequency of undamped $\left(Q^{-1}=0\right)$, harmonic $(\beta=0)$, unforced $\left(f_{0}=0\right)$ oscillations is equal to one. The quality factor $Q$ gives the ratio between the typical damping time and the oscillation period or, equivalently, between the width of the resonance curve and the oscillation frequency- and $\beta$ weights the relative strength of the nonlinear forcing. Clampedclamped oscillators have $\beta>0$, so that the nonlinearity hardens the total force.

The right-hand side of Eq. (1) stands for the self-sustaining force provided by the feedback circuit, as described in the Introduction. Here, $\phi(t)$ is the phase associated to the oscillatory motion, while $\phi_{0}$ and $f_{0}$ are the phase shift and the amplitude fixed by signal conditioning (see Section 1). For harmonic oscillations, the phase is defined in terms of the coordinate, from the identity $x(t)=A \cos \phi(t)$. 
For other kinds of motion, the phase can be defined in a variety of ways [13]. Elsewhere, we have discussed analytical and numerical definitions which are especially adapted to our specific problem [14]. In the present contribution, however, we deal mainly with harmonic motion.

Assuming that the self-sustained system performs oscillations with constant amplitude and frequency, the simplest approximation to handle the nonlinear effects of the cubic term is to disregard higher-harmonic contributions to the oscillatory motion -as traditionally done for the forced Duffing oscillator [3]. This corresponds to the lower-order approximation in the harmonic balance procedure [15]. To ease the comparison with the standard forced case, where the right-hand side of Eq. (11) is replaced by an oscillatory function of prescribed frequency, we write $\phi+\phi_{0} \equiv \Omega t$, so that all phases are measured from the initial $(t=0)$ phase of the self-sustaining force. We propose a harmonic solution $x(t)=A \cos \phi \equiv A \cos \left(\Omega t-\phi_{0}\right)$ and neglect higher-harmonic terms by approximating $\cos ^{3} \phi=\frac{3}{4} \cos \phi+\frac{1}{4} \cos 3 \phi \approx \frac{3}{4} \cos \phi$. Separating terms proportional to $\cos \phi$ and $\sin \phi$ yields the algebraic equations

$$
\left(1-\Omega^{2}\right) A+\tilde{\beta} A^{3}=f_{0} \cos \phi_{0}, \quad Q^{-1} \Omega A=f_{0} \sin \phi_{0},
$$

with $\tilde{\beta}=\frac{3}{4} \beta$. These equations can be brought to a third-degree polynomial relations between the unknowns, and are therefore explicitly solvable. Figures $2 \mathrm{a}$ and b show, as solid lines, the solutions for the amplitude $A$ and the frequency $\Omega$ as functions of the phase shift $\phi_{0}$, with $Q^{-1}=0.1, \tilde{\beta}=0.1$, and $f_{0}=1$. In Fig. 2re, the graph of amplitude versus frequency yields the typical Duffing resonance curve. Over this curve, $\phi_{0} \approx 0$ and $\pi$ at the leftmost and rightmost ends, respectively. At the peak, where the oscillator's response to the self-sustaining force is maximal, we 

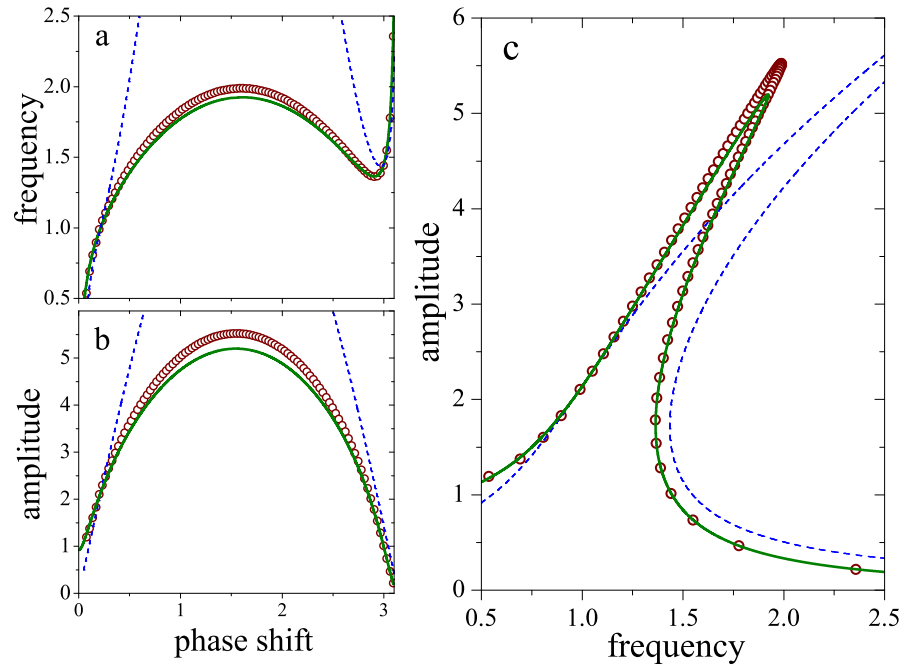

Fig. 2: Interdependence of amplitude, frequency, and phase shift for periodic motion of a forced Duffing oscillator, with $Q^{-1}=0.1, \tilde{\beta}=0.1$, and $f_{0}=1$. Solid lines: solutions to Eqs. (2); dotted lines: solutions to Eqs. (9); open dots: longtime numerical solutions to the equation of motion of the self-sustained oscillator, Eq. (1), for numerous values of the phase shift $\phi_{0}$.

have $\phi_{0}=\pi / 2$. For this phase shift, in fact, the force and the velocity $\dot{x}$ oscillate in-phase.

It is important to realize that the functional interdependence between $A, \Omega$, and $\phi_{0}$, given by Eqs. (2), is exactly the same whether the oscillator is subject to the self-sustaining force that fixes the phase shift $\phi_{0}$, or whether it moves under the action of an external force of given frequency $\Omega$. In particular, the resonance curve in Fig. 2r is the same irrespectively of the control parameter being the phase shift or the frequency. In the latter case, it is well known that, within the frequency range where three solutions for the amplitude exist, the two outermost solutions are stable, while the inner solution is unstable [3,16]. On the other hand, the 
frequency and amplitude plots in Fig. $2 \mathrm{a}$ and b suggest that, as the phase shift is varied, no bifurcations take place, so that stability should not change. In the next section, we show that -in contrast with the standard forced Duffing oscillatorperiodic motion under the action of a self-sustaining force with phase-shift control is in fact stable over the whole resonance curve.

\section{Stability under phase-shift control}

Whether the steady oscillations described by Eqs. (2) are stable, and represent the asymptotic motion of the Duffing oscillator, can be decided by a variety of perturbation techniques which, as a byproduct, provide an approximate solution to Eq. (11) beyond stationary harmonic oscillations. In our case, a convenient approach is provided by the method of multiple time scales [16, which -as demonstrated below- allows the oscillation frequency to vary in response to the control of the phase shift, and vice versa. This method discerns between a scale typical of the oscillatory motion, with period of order unity, and a longer time scale characteristic of slow changes in amplitude and frequency. It thus requires that all forces acting on the oscillator, apart from the linear elastic interaction, are treated as perturbations. To such end, we rewrite Eq. (11) as

$$
\ddot{x}+x=-\epsilon\left[Q^{-1} \dot{x}+\beta x^{3}-f_{0} \cos \left(\phi+\phi_{0}\right)\right],
$$

with $\epsilon$ the perturbation parameter. We write again $\phi+\phi_{0} \equiv \Omega t$ and, moreover, take $\Omega=1+\epsilon \omega$. Note that, while for the standard forced Duffing oscillator the phase difference between oscillation and forcing varies with time as the system approaches its asymptotic motion, in the self-sustained oscillator this phase shift is fixed and it is the oscillation frequency (i.e., $\omega$ ) which changes as time goes on. 
The method of multiple scales assumes that the solution to Eq. (30), $x(t ; \epsilon)$, depends on time through two auxiliary variables, $\tau_{0} \equiv t$ and $\tau_{1} \equiv \epsilon t$, respectively representing the fast and slow scales, and that it can be expanded as $x(t ; \epsilon)=$ $x_{0}\left(\tau_{0}, \tau_{1}\right)+\epsilon x_{1}\left(\tau_{0}, \tau_{1}\right)+\cdots$. Inserting this ansatz, and taking into account that $\frac{d}{d t}=\frac{\partial}{\partial \tau_{0}}+\epsilon \frac{\partial}{\partial \tau_{1}}$, zeroth and first-order terms in $\epsilon$ yield

$$
\frac{\partial^{2} x_{0}}{\partial \tau_{0}^{2}}+x_{0}=0
$$

and

$$
\frac{\partial^{2} x_{1}}{\partial \tau_{0}^{2}}+x_{1}=-2 \frac{\partial^{2} x_{0}}{\partial \tau_{0} \partial \tau_{1}}-Q^{-1} \frac{\partial x_{0}}{\partial \tau_{0}}-\beta x_{0}^{3}+f_{0} \cos \left(\tau_{0}+\omega \tau_{1}\right)
$$

respectively. Here, we have used the fact that $\Omega t=\tau_{0}+\omega \tau_{1}$. The (real) solution to Eq. (4) is

$$
x_{0}\left(\tau_{0}, \tau_{1}\right)=\frac{1}{2} a\left(\tau_{1}\right) \exp \left(i \tau_{0}\right)+\text { c.c. }=\operatorname{Re}\left[a\left(\tau_{1}\right) \exp \left(i \tau_{0}\right)\right]
$$

i.e. an oscillation modulated by a slowly varying amplitude. This solution must now be replaced into Eq. (5) to find $x_{1}\left(\tau_{0}, \tau_{1}\right)$. The solution for $x_{1}$, however, contains terms which grow indefinitely as time elapses. These secular contributions disappear if the following condition is required to hold:

$$
\frac{1}{2} \tilde{\beta} a^{2} a^{*}+i\left(a^{\prime}+\frac{1}{2} Q^{-1} a\right)=\frac{1}{2} f_{0} \exp \left(i \omega \tau_{1}\right)
$$

where the prime denotes differentiation with respect to $\tau_{1}$, and $a^{*}$ is the complex conjugate of $a$. This condition amounts to a differential equation to be satisfied by the complex amplitude $a\left(\tau_{1}\right)$. Writing $a=A \exp (i \alpha)$, where $A$ and $\alpha$ are real functions of $\tau_{1}$, we get

$$
\begin{aligned}
2 A^{\prime} & =-Q^{-1} A+f_{0} \sin \left(\omega \tau_{1}-\alpha\right), \\
2 A \alpha^{\prime} & =\tilde{\beta} A^{3}-f_{0} \cos \left(\omega \tau_{1}-\alpha\right) .
\end{aligned}
$$


Note that, having written $a=A \exp (i \alpha)$, the zeroth-order solution for $x(t)$ - given by Eq. (6) - can be cast as $x_{0}=A \cos \left[\Omega t-\left(\omega \tau_{1}-\alpha\right)\right]$. Thus, within this approximation, the combination $\psi=\omega \tau_{1}-\alpha$ is nothing but the phase shift between the coordinate and the forcing. For the standard forced Duffing oscillator, $\psi$ varies with time along with the oscillation amplitude $a$, while the frequency of the external force, $\Omega=1+\epsilon \omega$, remains constant. In this case, Eqs. (8) govern the evolution of $a$ and $\psi$ through the relation $\psi^{\prime}=\omega-\alpha^{\prime}$. Linearization of the equations yields the stability properties of their equilibrium solutions. In particular, this procedure shows the possible existence of the bistability frequency range commented on at the end of Section 2 (see Fig. 25) [16].

For the self-sustained oscillator, on the other hand, $\psi$ coincides with the fixed phase shift $\phi_{0}$ determined by the feedback circuit. Therefore, $\psi^{\prime}=\omega^{\prime} \tau_{1}+\omega-\alpha^{\prime}=0$, and Eqs. (8) transform into equations for the amplitude $a$ and the frequency $\omega$ :

$$
\begin{aligned}
2 A^{\prime} & =-Q^{-1} A+f_{0} \sin \phi_{0}, \\
2 A \omega^{\prime} & =\tau_{1}^{-1}\left(\tilde{\beta} A^{3}-f_{0} \cos \phi_{0}-2 A \omega\right) .
\end{aligned}
$$

The stationary solutions to these equations coincide with those of Eqs. (2) up to a term of order $\epsilon$ in the frequency. They are plotted in Fig. 2 as dotted lines. The substantial difference with the solutions to Eqs. (2), plotted as solid lines, is to be ascribed to the fact that, for the chosen values of $Q^{-1}, \tilde{\beta}$, and $f_{0}$, the effects of damping, nonlinearities, and external forcing are actually sizable additions to the linear elastic force, instead of small perturbations.

To avoid the singularity at $\tau_{1}=0$ in the second of Eqs. (9), we integrate the equations from $\tau_{1}=\tau_{i}>0$. The solutions are

$$
A\left(\tau_{1}\right)=Q f_{0} \sin \phi_{0}+\left[A\left(\tau_{i}\right)-Q f_{0} \sin \phi_{0}\right] \exp \left[-\left(\tau_{1}-\tau_{i}\right) / 2 Q\right]
$$




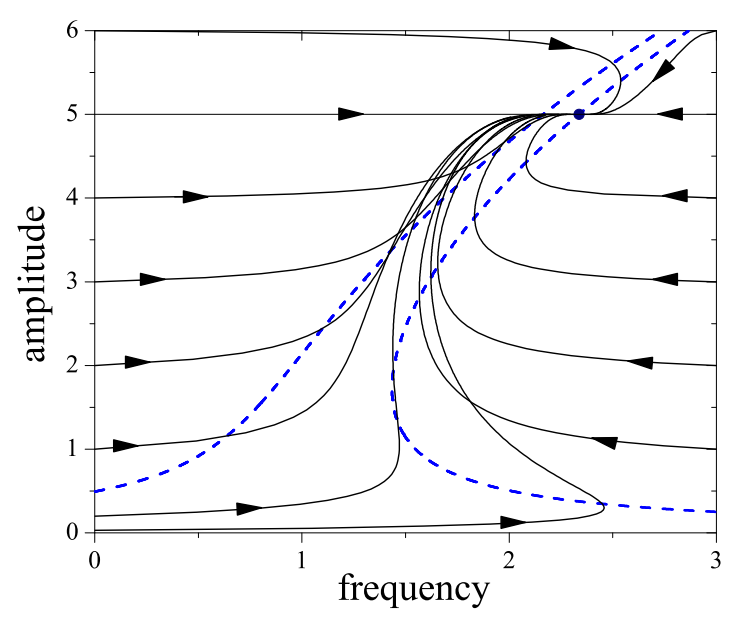

Fig. 3: The solutions to Eqs. (9) plotted in the $(\Omega, A)$-plane, for $Q^{-1}=0.1$, $\tilde{\beta}=0.1, f_{0}=1$, and $\tau_{i}=1$. The phase shift for the self-sustaining force is fixed to $\phi_{0}=\frac{5}{6} \pi$. Different lines correspond to different initial conditions. The full dot stands for the position of the equilibrium state, $\left(\frac{9}{4}+\frac{1}{20} \sqrt{3}, 5\right) \approx(2.337,5)$, which lies on the intermediate-amplitude branch of the resonance curve (dashed line).

and

$$
\omega\left(\tau_{1}\right)=\tau_{1}^{-1}\left[\tau_{i} \omega\left(\tau_{i}\right)+\int_{\tau_{i}}^{\tau_{1}} W(\tau) d \tau\right]
$$

with $W(\tau)=\frac{1}{2} A^{-1}(\tau)\left[\tilde{\beta} A^{3}(\tau)-f_{0} \cos \phi_{0}\right]$. Lines with arrows in Fig. 3 show these solutions in the frequency-amplitude plane, for various initial conditions, with $Q^{-1}=0.1, \tilde{\beta}=0.1, f_{0}=1$, and $\tau_{i}=1$. Note that, because of the dependence of the right-hand side of the second of Eqs. (9) on $\tau_{1}$, the trajectories can cross each other. The phase shift, $\phi_{0}=\frac{5}{6} \pi$, has been chosen in such a way that the corresponding equilibrium state lies on the branch of the resonance curve where the standard forced Duffing oscillator is unstable. For the self-sustained oscillator, in contrast, this equilibrium is clearly stable. 
Indeed, Eqs. (10) and (11) show that the equilibria of the self-sustained Duffing oscillator are stable for any value of the phase shift $\phi_{0}$, i.e. along the whole resonance curve. Moreover, for each value of $\phi_{0}$, the corresponding equilibrium attracts the trajectories starting at any initial condition and, hence, it is globally stable. The final approach to the equilibrium state is exponential in time for the amplitude, while it behaves as $\tau_{1}^{-1}$ for the frequency. The much slower long-time dependence for the frequency explains the flat asymptotic profile of the trajectories as seen in Fig. 3

The validity of the stability analysis based on Eqs. (9) is in principle limited to the perturbative limit, $\epsilon \rightarrow 0$. To decide whether the self-sustained Duffing system exhibits stable oscillations for any value of the phase shift beyond that limit, we implemented a numerical integration of the equation of motion (1). While the equation can be solved by standard techniques -in our case, the second-order RungeKutta algorithm- the numerical definition of the phase $\phi$ in the self-sustaining force requires extending the notion of phase to non-oscillatory behavior. In fact, during the numerical computation of $x(t)$ it cannot be assumed that the motion is always a harmonic oscillation. In a previous publication [14, we have given details on a suitable method to assign an instantaneous phase $\phi(t)$ to any form of $x(t)$, by locally fitting a trigonometric function. Dots in Fig. 2 show long-time numerical measurements of amplitude and frequency for $Q^{-1}=0.1, \tilde{\beta}=0.1, f_{0}=1$, and numerous values of $\phi_{0}$, ranging from $\phi_{0} \approx 0$ to $\pi$ at intervals of $0.01 \pi$. As in the perturbative limit, the solutions over the resonance curve are stable for all $\phi_{0}$. The difference with the perturbative stationary solution (dashed line) shows that we are well beyond that limit. On the other hand, the solution to Eqs. (2) (solid line) -obtained within the traditional approximation of nonlinearities- gives 
a very satisfactory description of the numerical results, except for the immediate vicinity of the peak.

\section{Internal resonance in a clamped-clamped oscillator}

As any other elastic body, the clamped-clamped beam has essentially an infinite number of oscillation modes. Nonlinear effects can couple these modes with each other, establishing an interaction in the form of mutual resonant excitation between different forms of oscillatory motion. Specifically, cubic nonlinearities make it possible that oscillations in the main mode synchronize with higher-harmonic modes whose frequency is three (or a multiple of three) times the fundamental frequency.

In a recent experiment [8], a silica clamped-clamped self-sustained microoscillator, about $500 \mu \mathrm{m}$ long, $3 \mu \mathrm{m}$ width, and $10 \mu \mathrm{m}$ thick, was shown to perform oscillations which, at very small amplitudes, had a frequency of approximately 66 $\mathrm{kHz}$. As the amplitude of the self-sustaining force was gradually increased by the experimenter, the amplitude of the oscillations increased as well, and-due to the hardening nonlinearity of the system-so did their frequency. When the frequency attained $68 \mathrm{kHz}$, however, it was observed that both the amplitude and the frequency ceased to grow, and both quantities reached a wide plateau where they remained practically constant. The self-sustaining force had to reach more than twice its amplitude at the beginning of the plateau for the oscillation amplitude and frequency to regain their growth.

The stabilization of the oscillation amplitude and frequency in the experiment was attributed to the resonant coupling between the main oscillation mode, sus- 
tained by the feedback circuit, and a higher-harmonic mode. In fact, finite-element numerical simulations of the clamped-clamped beam showed the existence of a torsional oscillation mode with a natural frequency slightly larger than three times the natural frequency of the main mode. As advanced in the Introduction, the technological interest of this phenomenon resides in the fact that an oscillator functioning in the resonant regime would maintain a very stable frequency, insensible to amplitude fluctuations in the self-sustaining force, thus providing a more reliable frequency reference [17. From a more fundamental perspective, an interesting aspect of this internal resonance is that nonlinearities play a twofold role in its origin. First, they induce the oscillation frequency to vary with the amplitude and, as a consequence, to reach the value where resonance is possible. Second, they are the source of the coupling between the main oscillation mode and higher harmonics.

In the experiment, the internal resonance was brought about by changing the amplitude $f_{0}$ of the self-sustained force. The phase shift in the feedback circuit, on the other hand, was kept constant at $\phi_{0} \approx \pi / 2$, i.e. close to the peak of the resonance curve, where the oscillator response to self-sustaining was maximal. Here, we show that the internal resonance can also be induced by varying the phase shift with fixed $f_{0}$. To this end, we implement a variation of the theoretical model used to explain the above described experimental results [8].

\subsection{Theoretical model}

In our description, following Sections 2 and 3 , the main oscillation mode is represented by a coordinate $x_{1}(t)$ satisfying the self-sustained Duffing equation (1). 
For the sake of simplicity, the higher-harmonic mode is represented by a coordinate $x_{2}(t)$ satisfying a linear unforced oscillator equation. The two equations are coupled to each other through linear interactions. Thus, for the main mode we have

$$
\ddot{x}_{1}+Q_{1}^{-1} \dot{x}_{1}+x_{1}+\beta x_{1}^{3}=f_{0} \cos \left(\phi+\phi_{0}\right)+J_{1} x_{2},
$$

while for the higher harmonic we have

$$
\omega_{2}^{-2} \ddot{x}_{2}+\omega_{2}^{-1} Q_{2}^{-1} \dot{x}_{2}+x_{2}=J_{2} x_{1},
$$

where $J_{1,2}$ are the coupling intensities, and $\omega_{2}$ is the higher-harmonic frequency (measured in units of the fundamental frequency; see Eq. (11)).

In order to focus on the phenomenon of frequency stabilization, we disregard the tripling of the frequency induced by the cubic nonlinearity, and concentrate on the synchronization of the two modes, assuming that their frequencies are similar. In other words, we take $\omega_{2} \gtrsim 1$. We thus look for solutions to Eqs. (12) and (13) where the two modes oscillate with the same frequency and their phases are locked to each other: $x_{1}(t)=A_{1} \cos \left(\Omega t-\phi_{0}\right), x_{2}(t)=A_{2} \cos \left(\Omega t-\phi_{2}\right)$. Proceeding as with Eq. (11), we find the following algebraic equations for the amplitudes $A_{1,2}$, the frequency $\Omega$ and the phase difference $\psi_{2}=\phi_{0}-\phi_{2}$ between the higher-harmonic oscillation and the main mode:

$$
\begin{aligned}
& \left(1-\Omega^{2}\right) A_{1}+\tilde{\beta} A_{1}^{3}=f_{0} \cos \phi_{0}+J_{1} A_{2} \cos \psi_{2}, \\
& Q_{1}^{-1} \Omega A_{1}=f_{0} \sin \phi_{0}+J_{1} A_{2} \sin \psi_{2}, \\
& \left(\omega_{2}^{2}-\Omega^{2}\right) A_{2} \cos \psi_{2}-\omega_{2} Q_{2}^{-1} \Omega A_{2} \sin \psi_{2}=\omega_{2}^{2} J_{2} A_{1}, \\
& \left(\omega_{2}^{2}-\Omega^{2}\right) A_{2} \sin \psi_{2}+\omega_{2} Q_{2}^{-1} \Omega A_{2} \cos \psi_{2}=0 .
\end{aligned}
$$

As in the case of Eqs. (2), these equations are equivalent to third-degree polynomial relations between the unknowns, and thus can be exactly solved. 

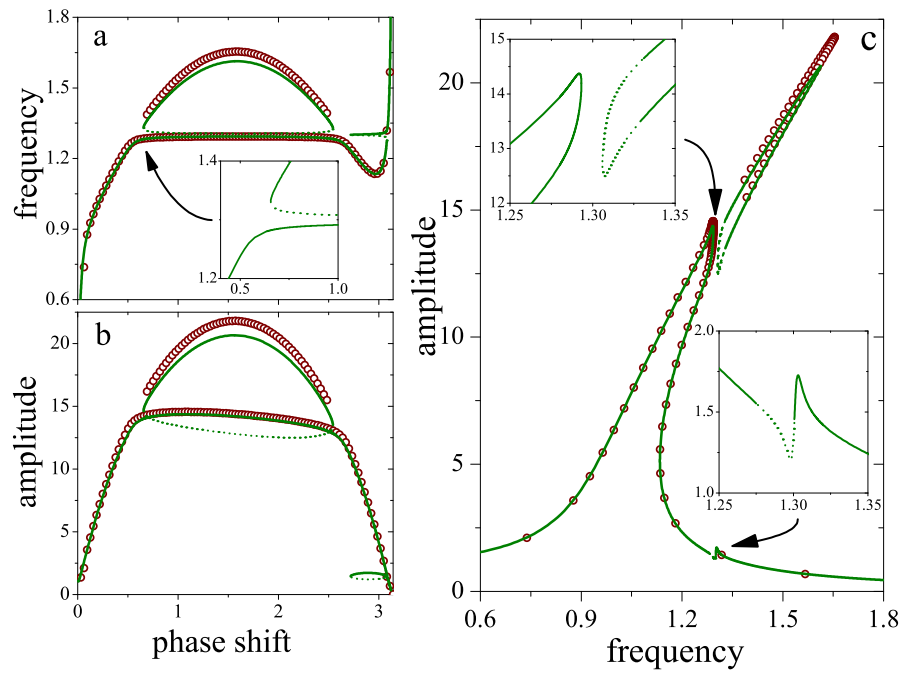

Fig. 4: Interdependence of the amplitude $A_{1}$ of the main oscillation mode, the synchronization frequency $\Omega$, and the self-sustaining phase shift $\phi_{0}$, for a forced Duffing oscillator coupled to a linear oscillator representing a higher-harmonic mode. Lines: solutions to Eqs. (14), with $\beta=0.005, f_{0}=1, \omega_{2}=1.3, Q_{1}^{-1}=0.03$, $\omega_{2}^{-1} Q_{2}^{-1}=0.003, J_{1}=10^{-4}$, and $J_{2}=1$. Solid and dotted sections represent stable and unstable oscillations, respectively. Open dots: long-time numerical solutions to Eqs. (12) and (13) for the same set of parameters, and numerous values of the phase shift $\phi_{0}$. The insets show close-ups of the zones pointed to by the arrows. For clarity, numerical results are not shown in the insets.

Curves in the three panels of Fig. 4 illustrate the interdependence between the amplitude $A_{1}$, the synchronization frequency $\Omega$, and the phase shift $\phi_{0}$, as determined by Eqs. (14), for $\beta=0.005, f_{0}=1, \omega_{2}=1.3, Q_{1}^{-1}=0.03, \omega_{2}^{-1} Q_{2}^{-1}=$ 0.003, $J_{1}=10^{-4}$, and $J_{2}=1$. Comparison with Fig. 2 makes it clear that the overall outline of the curves is the same as for the self-sustained Duffing oscillator considered in Section 3. However, a crucial feature shows up for synchronization 
frequencies around the higher-harmonic frequency $\omega_{2}$. In the close-ups of Fig. 4 r, we see that the resonance curve develops a gap across the direction of the frequency axis, so that the peak is cut off from the rest of the curve in a kind of elongated "island." For frequencies within the gap, the only possible solution lies on the low-amplitude branch, where the curve acquires in turn an up-down peak whose center and width coincide with those of the gap.

In terms of the variation of the control parameter $\phi_{0}$, in Fig. 4 , we see that only one solution exists for small phase shifts, with a rapidly increasing frequency. As $\phi_{0}$ grows further and the frequency approaches that of the higher-harmonic mode, however, the growth of the frequency flattens abruptly and, at the same time, two new solutions appear. Of these two new solutions, the one with the lower frequency remains close to the preexisting solution, with which it determines the frequency gap referred to in the preceding paragraph. Eventually, well beyond the central part of the curves, the two additional solutions disappear and the frequency of the preexisting solution decreases. Note the sizable interval of phase shifts $\left(0.6 \lesssim \phi_{0} \lesssim 2.6\right.$ for the parameters of Fig. 4) along which the preexisting solution maintains its frequency at a practically constant level. For all these values of our control parameter, the main-mode frequency is thus strongly stabilized by the internal resonance. A small additional interval with three solutions, where the frequency is again stabilized, is found for larger values of the phase shift $\left(2.7 \lesssim \phi_{0} \lesssim 3\right)$ in correspondence with the up-down peak of the resonance curve.

From the third and fourth of Eqs. (14) it can be immediately seen that the amplitude of the higher-harmonic mode is

$$
A_{2}=\frac{\omega_{2}^{2} J_{2} A_{1}}{\sqrt{\left(\omega_{2}^{2}-\Omega^{2}\right)^{2}+\omega_{2}^{2} Q_{2}^{-2} \Omega^{2}}} .
$$


As expected for a linear oscillator, this amplitude is proportional to the forcing amplitude $J_{2} A_{1}$, but is also modulated by a frequency-dependent Lorentzian factor. This modulation implies that $A_{2}$ attains significant values only when the synchronization frequency $\Omega$ reaches the vicinity of the higher-harmonic frequency $\omega_{2}$, i.e. around the frequency gap. Equation (15) also shows that the width of this zone is $\Delta \Omega \sim \omega_{2} Q_{2}^{-1}$, and is therefore inversely proportional to the quality factor of the higher-harmonic mode. It is within this zone that the coupling between the two modes is most effective, and resonant energy transfer takes place from the self-sustained main mode.

Taking into account the results presented in Section 3, the stability of the periodic solutions given by Eqs. (14) can be inferred from a standard analysis of the plot in Fig. 4a, which we interpret as a bifurcation diagram for the frequency as a function of the phase shift $\phi_{0}$. Assuming that the solution branches present in both Figs. 2 and 4 have the same stability properties, we expect that the only solution found in Fig. $4 \mathrm{a}$ for small phase shift is stable. The appearance of two new solutions as the phase shift grows should be associated with a saddle-node bifurcation, creating a pair of solutions with opposite stability. The upper branch, also present in Fig. 2a, should correspond to the stable one. Upon further increasing of $\phi_{0}$, an inverse saddle-node bifurcation annihilating the same pair takes place and, a little farther, a new saddle-node bifurcation gives rise to the two solutions in the small peak. The unstable solution of this new pair annihilates in turn with the preexisting stable solution, while its stable partner subsists until the phase shift attains its largest values, constituting the rightmost branch of the bifurcation diagram. 


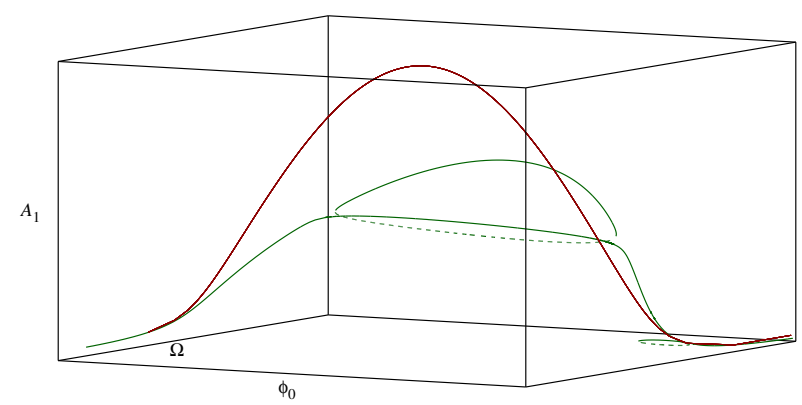

Online Resource 1 (ESM_1.gif): Animation with rotating three-dimensional visualization of the internal-resonance curve in the $\left(\phi_{0}, \Omega, A_{1}\right)$-space, as given by the solution to Eqs. with the parameters of Fig. 4 The resonance curve for a forced linear oscillator with the same $Q$ and $f_{0}$ is plotted for comparison.

We have verified these conclusions on the stability properties of the periodic solutions by numerically solving the equations of motion, with the same scheme as in Section 3 Dots in Fig. 4 show long-time numerical measurements of amplitude and frequency with the same parameter choice as for the curves. The control parameter $\phi_{0}$ varies from $\phi_{0} \approx 0$ to $\pi$ at intervals of $0.01 \pi$. In the zones where three solutions exist, we have integrated the equations of motion at least twice, starting from different initial conditions, compatible with large and small oscillation amplitudes. In the small interval corresponding to the up-down peak, we have refined the phase-shift sampling to get better evidence on the stability of the three solutions. For the sake of clarity, numerical results in this interval are not included in Fig. 4 .

The bifurcation scenario is clearly depicted by the plot of frequency vs. phase shift in Fig. 4a (see inset), but may turn out to be less obvious in the plot of amplitude vs. phase shift in Fig. $4 \mathrm{~b}$, or in the resonance curve. In order to ease the 
visualization of the whole picture, the Online Resource 1 contains an animation with views in the three-dimensional space spanned by the coordinates $\left(\phi_{0}, \Omega, A_{1}\right)$, which illustrates how the three main plots of Fig. 4 connect with each other.

4.2 Preliminary experimental results and the backbone approximation

As mentioned above, previous experiments on frequency stabilization by internal resonance in clamped-clamped microoscillators were carried out for fixed phase shift $\left(\phi_{0} \approx \pi / 2\right)$ and varying the amplitude of the self-sustaining force [8]. Under these conditions, due to the effect of the cubic force, the oscillation frequency increases with the amplitude until it reaches the resonance region. The results of Section 4.1, in turn, show that the resonance can also be induced by keeping the self-sustaining amplitude fixed and varying the phase shift.

We have performed preliminary measurements under phase-shift control on the same kind of self-sustained microoscillators as used in previous experiments. The phase shift is tuned by means of a variable resistor in an all-pass filter intercalated in the self-sustaining feedback circuit. Details of the electronics can be found elsewhere [18]. Dots in the main plot of Fig. [5] stand for our measurements in the amplitude-frequency plane, i.e. over the resonance curve. The amplitude is measured from an oscillating electric signal produced by the vibrating silica bar through a capacitive transducer, and is therefore given in millivolts. The uncertainty in the determination of amplitude and frequency was around $0.015 \mathrm{mV}$ and $0.1 \mathrm{kHz}$, respectively.

As the phase shift is increased from small values, first, the frequency and the amplitude grow as well. Then, when the frequency reaches some $63.3 \mathrm{kHz}$, the 


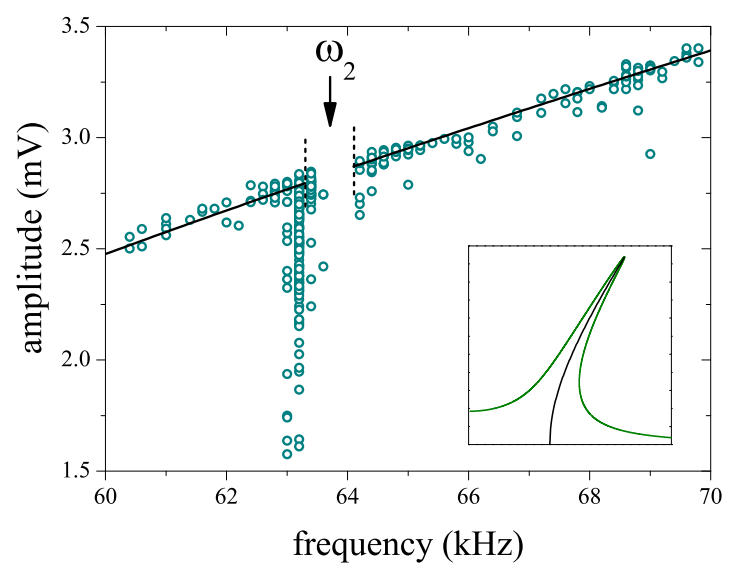

Fig. 5: Experimental measurements (dots) of the amplitude $A_{1}$ and frequency $\Omega$ of the main oscillation mode under phase-shift control of a self-sustained clampedclamped microoscillator. Experimental errors (not displayed) were estimated in $0.015 \mathrm{mV}$ for the amplitude and $0.1 \mathrm{kHz}$ for the frequency. Vertical dashed segments indicate the frequency gap caused by the internal resonance, around $\omega_{2} \approx 63.7 \mathrm{kHz}$. The solid line is a fit of the experimental data with the backbone approximation to our analytical model. The inset illustrates the backbone approximation for a resonance curve with the parameters of Fig. 2 ( $Q=10)$. The approximation improves sharply as $Q$ grows.

oscillations suddenly become irregular, with their amplitude varying erratically over a rather wide interval. The frequency, on the other hand, remains stable at a rather well-defined value, insensible to the amplitude fluctuations. Frequency stabilization is apparent at this point. Further increase of the phase shift leads again to more stable oscillations, regaining the regime where both the amplitude and the frequency grow with $\phi_{0}$. A gap, however, has been left vacant for frequencies 
between 63.3 and $64.1 \mathrm{kHz}$, corresponding to amplitudes between 2.8 and $2.9 \mathrm{mV}$, approximately.

Our experimental results can be satisfactorily fitted by the model presented in Section 4.1, with a suitable choice of its parameters. To this end, it is useful to note first that the quality factor of a clamped-clamped silica microoscillator as used in the experiments is typically in the order of $10^{4}[\underline{8}$. This large quality factor implies that the Duffing resonance curve is very narrow, with its two leaning branches very close to each other. Under these conditions, the two branches are well represented by a single curve -sometimes called backbone curve [16]-given by an approximate expression for the interdependence between amplitude and frequency. The inset of Fig. 5 shows a resonance curve with $Q=10$ and its backbone approximation. As $Q$ grows and the curve becomes narrower, the approximation is increasingly good.

The backbone approximation for the Duffing resonance curve is obtained from Eqs. (2) by neglecting the contribution of damping and of the external force. Thus, it corresponds to assuming not only a large quality factor but also a large oscillation amplitude. With the notation of Eqs. (2), the backbone curve is given by $A=\sqrt{\left(\Omega^{2}-1\right) / \tilde{\beta}}$. The value of the phase shift $\phi_{0}$ along the curve can then be determined from the second of those equations: $\sin \phi_{0}=\Omega A / Q f_{0}$. In order to apply this approximation to fit the experimental data shown in Fig. 5, we first disregard the effect of the internal resonance, and write

$$
A_{1}=a_{1} \sqrt{\left(\Omega / \omega_{1}\right)^{2}-1}
$$

where $a_{1}$ and $\omega_{1}$ are tunable parameters, respectively given by the units of measure of $A_{1}$ and $\Omega$. Our estimate yields $a_{1}=(6.4 \pm 0.1) \times 10^{-2} \mathrm{mV}$, and $\omega_{1}=(46 \pm 1)$ $\mathrm{kHz}$. The line in the main plot of the figure stands for this estimate. Assuming for 
our microoscillator a quality factor of the order of $10^{4}$, the width of the resonance curve would fall well inside the dispersion of the experimental data, which justifies using the backbone approximation.

To fit the gap in the backbone curve, indicated by vertical dashed lines in Fig. 5. we now turn the attention to Eqs. (14). Solving the two last equations for the product $A_{2} \sin \psi_{2}$, and replacing the result into the second equation, makes it possible to write

$$
\sin \phi_{0}=\left[1+\frac{\nu^{4}}{\left(\omega_{2}^{2}-\Omega^{2}\right)^{2}+\omega_{2}^{2} Q_{2}^{-2} \Omega^{2}}\right] \sin \Phi_{0} .
$$

The constant $\nu^{4}$-where $\nu$ has units of frequency- is proportional to the product of the coupling constants, $J_{1} J_{2}$, and to the ratio of the quality factors of the two involved oscillation modes, $Q_{1} / Q_{2}$. These quantities cannot be discerned from each other in our experiment, and $\nu$ must therefore be considered as a single fitting parameter. The angle $\Phi_{0}$, in turn, can be associated to the phase shift in the zone of the gap when the internal resonance is absent, i.e. for $\nu^{4} \propto J_{1} J_{2}=0$. From our measurements of amplitude and frequency as functions of the phase shift (not presented here) we estimate $\sin \Phi_{0}=0.16 \pm 0.01$.

Equation (17) makes it clear that the gap in the backbone curve appears when the second term inside the square bracket in the right-hand side is large enough as to make $\sin \phi_{0}>1$, for which no real value of the phase shift $\phi_{0}$ satisfies the equation. The Lorentzian profile of this term as a function of the frequency $\Omega$ indicates that the gap occurs around $\omega_{2}$. As advanced in Section 4.1, its width $\Delta \Omega$ is proportional to $\omega_{2} Q_{2}^{-1}$. Locating the center of the gap, we estimate $\omega_{2}=$ $(63.7 \pm 0.2) \mathrm{kHz}$. Tuning the constant $\nu$, on the other hand, requires knowing the quality factor $Q_{2}$ of the higher-harmonic mode, to which we do not have access 
in the experiment. However, if -in the spirit of the backbone approximation- we assume that $Q_{2}$ is large, the contribution of the term $\omega_{2}^{2} Q_{2}^{-2} \Omega^{2}$ in the denominator of the Lorentzian function can be neglected by comparison to $\left(\omega_{2}^{2}-\Omega^{2}\right)^{2}$. Within this assumption, we find $\nu^{4}=\left(\omega_{2} \Delta \Omega\right)^{2}\left(1-\sin \Phi_{0}\right) / \sin \Phi_{0}$. Taking $\Delta \Omega=0.8 \mathrm{kHz}$, our estimate yields $\nu=(10.8 \pm 0.2) \mathrm{kHz}$.

\section{Conclusion}

Micromechanical devices have opened the possibility of renewed mutual contribution between nonlinear physics and technological applications [7, 19]. On one side, this minute machines -still belonging to the realm of Newtonian mechanicsoften function within regimes where nonlinear effects play a substantial role in the dynamics. This is particularly true for micromechanical oscillators, which are foreseen to be used as pacemakers in time-keeping electronic devices, and whose large vibration amplitudes bring them well beyond the linear elastic regime. On the other side, well-developed techniques of MEMS fabrication can be used to build up microscale lab equipment, where complex physical phenomena such as the collective dynamics of large populations of nonlinear coupled oscillators may be realized and tested. This path is just beginning to be explored [20,21].

In this paper, we have studied two technologically relevant aspects of the selfsustained Duffing oscillator, which models a clamped-clamped elastic bars inserted in a feedback circuit. Firstly, following the traditional multiple-scale approach used to study the stability of the standard Duffing equation [16], we have shown that the self-sustained oscillator is stable along the entire resonance curve. This result contrasts with the well-known standard behavior, where an unstable branch devel- 
ops in the middle part of the curve. The difference resides in the fact that, in the self-sustained oscillator, the system is controlled by fixing the phase shift between oscillation and forcing whereas, in the standard case, the control parameter is the forcing frequency. The situation is reminiscent -although not fully equivalent- to the basic process of feedback stabilization prescribed by control theory. We have shown that, within the multiple-scale approximation, the equations of motion can be exactly solved, and provided numerical evidence that validate the approximation.

In the second place, we have elaborated on a model for the internal resonance between the main oscillation mode and a higher-harmonic mode in a clampedclamped oscillator. The interest of this phenomenon lies in that it has been associated with the frequency stabilization observed in microoscillators upon variation of the forcing amplitude [8]. Frequency stability under amplitude fluctuations is an unavoidable condition for the use of microoscillators as frequency references in time-keeping devices. The model describes the internal resonance as a synchronization process between the main nonlinear oscillation, represented by the selfsustained Duffing equation, and a linear oscillator of different frequency coupled to the main mode. Due to this phenomenon, the Duffing resonance curve develops a gap, which is associated to a wide phase-shift interval where the oscillation frequency remains practically constant. As similar "island" effect has recently been reported for an externally forced Duffing oscillator coupled through displacement and velocity to a linear oscillator $[22$. It is interesting to point out that, in the vicinity of the gap, the amplitude-frequency interdependence is similar to that of the standard forced Duffing oscillator at its subharmonic resonances [16]. In order to establish the stability properties of the model, we have performed numer- 
ical simulations. These support a picture where the resonance occurs through two pairs of direct-inverse saddle-node bifurcations. Finally, we have presented preliminary experimental results which illustrate the internal resonance in the situation where, contrary to previous experiments, the phase shift between forcing and oscillation was controlled. Model and experiment were fitted to each other within a high-quality-factor approximation.

At the microscale, an important dynamical ingredient - that has however been purposely left aside in our description- is added by noise. Random fluctuations of both thermal and electronic origin become increasingly influential on the mechanics of micromachines as these decrease in size. In the case of oscillators, noise makes it necessary to work at larger amplitudes, which in turn increases the effects of nonlinearities. To our knowledge, a theoretical approach to the interplay between noise and nonlinearities focused on the dynamics of micromechanical systems is still lacking. This seems to be an appealing next step in the lines of the present research, where the physics of stochastic processes should have their say.

Acknowledgements We acknowledge financial support from ANPCyT (PICT 2011-0545), Argentina, and enlightening discussions with Hernán Pastoriza, Darío Antonio, and Daniel López. Experiments were conducted at the MEMS Laboratory of Centro Atómico Bariloche.

\section{References}

1. G. Duffing, Erzwungene Schwingungen bei Veränderlicher Eigenfrequenz. F. Vieweg u. Sohn, Braunschweig (1918)

2. I. Kovacic, M. J. Brennan, The Duffing Equation: Nonlinear Oscillators and Their Behaviour. Wiley, New York (2011) 
3. L. D. Landau, E. M. Lifshitz, Mechanics. Course of Theoretical Physics, Vol. 1. ButterworthHeinemann, Oxford (1976)

4. P. J. Holmes, D. A. Rand, The bifurcations of Duffing's equation: An application of catastrophe theory, J. Sound Vibr., 44, 237-253 (1976)

5. P. J. Holmes, A nonlinear oscillator with a strange attractor, Phil. Trans. Royal Soc. A, 292, 419-448 (1979)

6. P. J. Holmes, D. Whitley, On the attracting set for Duffing's equation, II: A geometrical model for moderate force and damping, Physica, 7D, 111-123 (1983)

7. K. L. Ekinci, M. L. Roukes, Nanoelectromechanical systems, Rev. Sci. Instrum., 76, 061101 (2005)

8. D. Antonio, D. H. Zanette, D. López, Frequency stabilization in nonlinear micromechanical oscillators, Nat. Comm., 3, 806 (2012)

9. D. K. Agrawal, J. Woodhouse, A. A. Seshia, Modelling nonlinearities in MEMS oscillators, IEEE Trans. Ultrason. Ferroelectr. Freq. Control, 60, 1646-1659 (2013)

10. R. Narashima, Nonlinear vibration of an elastic string, J. Sound Vib., 8, 134-155 (1968)

11. T. C. Molteno, N. B. Tufillaro, An experimental investigation into the dynamics of a string, Am. J. Phys., 72, 1157-1167 (2004)

12. B. Yurke, D. S. Greywall, A. N. Pargellis, P. A. Busch, Theory of amplifier-noise evasion in an oscillator employing a nonlinear resonator, Phys. Rev. A, 51, 4211-4229 (1995)

13. Y. Kuramoto, Chemical Oscillations, Waves, and Turbulence. Springer, Berlin (1984)

14. S. I. Arroyo, D. H. Zanette, Synchronization properties of self-sustained mechanical oscillators, Phys. Rev. E, 87, 052910 (2013)

15. T. Detroux, L. Renson, G. Kerschen, The harmonic balance method for advanced analysis and design of nonlinear mechanical systems, in G. Kerschen, ed., Nonlinear Dynamics, Vol. 2. Conference Proceedings of the Society for Experimental Mechanics Series, Chapter 3. Springer, Berlin (2014)

16. A. H. Nayfeh, D. T. Mook, Nonlinear Oscillations. Wiley, New York (1995)

17. M. Agarwal, S. A. Chandorkar, H. Mehta, R. N. Candler, B. Ki, M. A. Hopcroft, A study of electrostatic force nonlinearities in resonant microstructures, Appl. Phys. Lett., 92, 104106 (2008) 
18. S. I. Arroyo, Dynamics of clamped-clamped oscillators. M. Sc. Thesis. http://ricabib.cab.cnea.gov.ar/449/ (2013)

19. L. G. Villanueva, R. B. Karabalin, M. H. Matheny, D. Chi, J. E. Sader, M. L. Roukes, Nonlinearity in nanomechanical cantilevers, Phys. Rev. B, 87, 024304 (2013)

20. D. K. Agrawal, J. Woodhouse, A. A. Seshia, Observation of locked phase dynamics and enhanced frequency stability in synchronized micromechanical oscillators, Phys. Rev. Lett., $111,084101(2013)$

21. M. H. Matheny, M. Grau, L. G. Villanueva, R. B. Karabalin, M. C. Cross, M. L. Roukes, Phase synchronization of two anharmonic nanomechanical oscillators, Phys. Rev. Lett., 112, $014101(2014)$

22. G. Habib, T. Detroux, R. Viguié, G. Kerschen, Nonlinear generalization of Den Hartog's equal-peak method. Mech. Sys. Sig. Proc., 52-53, 17-28 (2015) 